\title{
Computerized tomography features and clinicopathological analysis of Kimura disease in head and neck
}

\author{
LI ZHANG $^{1}$, LI YAO ${ }^{2}$, WEI-WEI ZHOU ${ }^{3}$, JIAN-NAN MA ${ }^{1}$ and CHUN-QIAN ZHANG ${ }^{1}$
}

Departments of ${ }^{1}$ CT Diagnosis, ${ }^{2}$ Cardiology and ${ }^{3}$ Pathology, Cangzhou Central Hospital, Cangzhou, Hebei 061001, P.R. China

Received November 28, 2017; Accepted May 2, 2018

DOI: $10.3892 /$ etm.2018.6431

\begin{abstract}
The aim of the current study was to discuss and analyze computerized tomography (CT) features and clinicopathological characteristics of patients with Kimura disease in head and neck. CT and clinicopathological data of 12 patients with Kimura disease diagnosed between May 2011 and May 2015 were analyzed. The mean age was 43.9 years and the disease course varied from 1 week-20 years. The mean absolute value of eosinophil granulocytes was $1.95 \times 10^{9} / 1$. Multiple nodules were detected in 4 patients with Kimura disease. Main symptoms included multiple enlarged lymph nodes in neck and submaxillary regions. CT imaging of nodular lesions revealed a clear boundary, with moderate to marked homogeneous enhancement. Diffuse mass lesions were observed in 6 patients with Kimura disease. Main symptoms included subcutaneous diffuse soft tissue mass in parotid gland and maxillofacial regions. CT imaging of diffuse mass revealed unclear boundaries, adjacent skin thickness and moderate heterogeneous enhancement. In some cases, the diffuse mass involved soft mass with unclear boundaries. Pathological symptoms of Kimura disease included infiltration of eosinophilia, lymphocytic proliferation, formation of lymphatic follicles and variable degrees of fibrosis and vascular proliferation. Diagnosis of Kimura disease in head and neck regions may be improved based on lesions with clear or unclear boundaries, homogeneous or heterogeneous enhancement, with or without lymphadenectasis and by the presence of peripheral blood eosinophilia. However, a final diagnosis relies on a pathological examination.
\end{abstract}

\section{Introduction}

Kimura disease is a rare lymphoproliferative disease of unknown origin that occurs in the head and neck (1). A majority

Correspondence to: Dr Li Zhang, Department of CT Diagnosis, Cangzhou Central Hospital, 16 Xinhua West Road, Cangzhou, Hebei 061001, P.R. China

E-mail: zhangli5475@163.com

Key words: Kimura disease, computerized tomography, clinicopathological examination of Kimura disease cases have been reported in China, Japan and other Southeast Asian countries and rarely in European countries $(1,2)$. The cause of Kimura disease is unclear, but it may be associated with autoimmunity, insect bites or infections and allergies caused by parasites (3). As Kimura disease often results in an increase of lesions, peripheral blood eosinophils and elevated serum immunoglobulin E ( $\operatorname{IgE}$ ) levels, it is considered the result of inflammation caused by immune dysfunction (4). IgE-mediated type I allergy caused by abnormal regulation of $\mathrm{CD}^{+}$cells may describe the major pathogenic mechanism (5).

A majority of reports on Kimura disease focus on clinical findings from China, Korea and Southeast Asian countries, with few computerized tomography $(\mathrm{CT})$ reports published $(3,4)$. Gopinathan and Tan (6) examined CT features of 13 patients with Kimura disease and divided Kimura disease into two categories: Type I and type II. Type I exhibited a clear boundary and marked homogeneous enhanced nodules. In the study, lesions were of uniform density and had clear boundaries, with moderate or marked homogeneous enhancement. The degree of enhancement in the arterial and venous phase was similar. Type I Kimura disease nodules had an intact capsule, pointing to lymph node swelling in the head and neck region, particularly in the parotid gland. Type II Kimura disease exhibited unclear boundaries around nodules and mild inhomogeneous enhancement with an uneven structure. In addition, increased hospitalization times were recorded for these patients. Gopinathan and Tan (6) reported $30.70 \%$ of cases with diffuse masses. Boundaries of these lesions were unclear, demonstrated an ambiguous fat layer and increased density. There was no obvious cystic degeneration, necrosis or calcification, and lesions exhibited moderate heterogeneous enhancement. No obvious capsules were observed in Type II nodules. In the study, boundaries of patients with type I and type II Kimura disease nodules and lesions were unclear. In these cases, lesions were characterized by a large amount of eosinophils and inflammatory cell infiltration inside and outside of capsules. Gopinathan and Tan (6) concluded that further studies are needed to determine whether an unclear boundary represented the transition from type I to type II Kimura disease nodules.

Clinical symptoms of Kimura disease include a painless soft tissue mass, with peripheral lymphadenopathy or lymphadenectasis in the neck and submandibular region (7). Kimura disease may be misdiagnosed as malignant tumors based on imaging and clinical symptoms (8). A precise preoperative diagnosis is important. Postoperative recurrence 
of Kimura disease is high to $62 \%$ (9). Kimura disease is sensitive to hormone therapy, surgery, radiotherapy, steroid therapy, intravenous immunoglobulin and cyclosporin A (6). In the current study, CT manifestations and clinical pathological features of patients with Kimura disease were analyzed retrospectively to improve the understanding of the disease.

\section{Materials and methods}

General information. Clinical, imaging and pathological data of 12 patients with Kimura disease (males, $n=11$; females, $\mathrm{n}=1$ ) diagnosed by postoperative pathology or open biopsy at Cangzhou Central Hospital (Cangzhou, China) between May 2011 and May 2015 were analyzed (Table I). Patients were aged from 26-71 years, with a mean age of 43.9 years. The course of the disease ranged from 1 week-20 years. There were 7 patients with unilateral disease and 5 patients with bilateral disease. A total of 10 patients held an initial diagnosis. Kimura disease recurred in 1 patient with a 20 -year history of the disease and 1 patient with a 5-year history of the disease. There were no known causes of unilateral or bilateral parotid, submandibular painless nodules or masses in the neck. In all cases, nodules had grown slowly following the onset of symptoms and initial diagnosis. Pruritus in the lesion area was exhibited in 1 patient with thickening of the skin surface and pigmentation. In all patients, blood eosinophilia exceeded $0.63 \times 10^{9} / 1$ [normal count, $0.02-0.52 \times 10^{9} / 1(10)$; Table I]. Terms were defined as follows: Soft, referring to lymphangioma or abscess; hard, referring to the hardness of bone tissue and medium, an intermediate stage.

CT examination. All patients underwent a CT scan and a contrast-enhanced scanning of the head and neck using a GE Light Speed 64-slice spiral CT scanner (tube voltage, $120 \mathrm{kV}$; tube current, $250 \mathrm{~mA}$; thickness, $5 \mathrm{~mm}$; layer spacing, $5 \mathrm{~mm}$; pitch, 1.375; conventional 2.5-mm thin-slice reconstruction). Ultravist was used for contrast-enhanced scanning. A high-pressure injector was used for intravenous bolus injection of the contrast agent, with an injection rate of $3.5 \mathrm{ml} / \mathrm{sec}$ through the elbow. The degree of enhancement of the lesions was judged by the difference in CT values. A value of 10-20 Hounsfield units (HU) was defined as mild enhancement and a value of 20-40 HU was considered moderate enhancement. Marked enhancement was at $>40 \mathrm{HU}$.

Pathological examination. Color of the masses, texture and borders were analyzed. Specimens were fixed with $4 \%$ neutral formalin solution at room temperature for $6 \mathrm{~h}$, followed by conventional dehydration, paraffin embedding and hematoxylin and eosin staining. The thickness of the sections was $4 \mu \mathrm{m}$. Sections were treated with $1 \%$ periodic acid solution for $10 \mathrm{~min}$ at room temperature, washed with PBS for $5 \mathrm{~min}$, treated with Schiff solution for $10 \mathrm{~min}$ and washed with PBS for further $10 \mathrm{~min}$. Section were stained with hematoxylin for $1 \mathrm{~min}$ at $60^{\circ} \mathrm{C}$. Sections were observed by light microscope (magnification, $\mathrm{x} 100$ ).

\section{Results}

CT features. Out of 12 patients with Kimura disease, 6 cases involved the parotid gland (3 unilateral and 3 bilateral),
12 cases involved lymphadenectasis, 1 case involved the rear of the right auricle and lymphadenectasis, 1 case involved the subcutaneous diffuse soft tissue mass of the left cheek and lymphadenectasis and 6 cases involved the parotid gland and lymphadenectasis (Table I).

Parotid gland lesions. Pathological tissues of parotid gland lesions were obtained from postoperative specimen $(n=4)$ or open biopsy $(n=2)$. The parotid gland volume was increased and a focal nodular or diffuse mass was observed. In 3 cases, multiple lesions were present. Lesions had a uniform density and clear boundaries, with moderate or marked homogeneous enhancement. The degree of enhancement in arterial and venous phases was similar (Fig. 1). A diffuse mass was present in 5 patients. The boundaries of the lesions were unclear, with an undefined fat layer and increased density. There was no visible cystic degeneration, necrosis or calcification and mild or moderate heterogeneous enhancement was observed (Fig. 2A). Interstitial fibrosis and hyaline degeneration of the capillary wall were further observed in Fig. 2A.

Cervical lymph node lesions. A total of 2 patients had varying numbers of cervical lymph node lesions in neck region. Pathological tissues of cervical lymph node lesions were obtained from the postoperative specimen. In patients with ipsilateral neck masses, the boundaries of the masses were clear and exhibited homogeneous enhancement, which was increased compared with cases of parotid lesions (Fig. 2B). Fig. 3 visualizes unclear boundaries of the lesions, with moderate or marked homogeneous enhancement. The short diameter of the largest lymph node was $23.9 \mathrm{~mm}$. Figs. 4 and 5 present the clear boundaries of lesions with a uniform density. There was no marked liquefaction necrosis, calcification or fusion, with moderate or marked homogeneous enhancement. There was 1 case of disease recurrence in a patient with a 10 -year history of the disease. The boundary of the parotid mass of the case of recurrence was unclear, with mild or moderate heterogeneous enhancement.

General pathological features. All lesions were soft or medium hard and the cut surface of the lesion was gray or gray-yellow. Lymph nodes had a complete capsule. In contrast, boundaries of parotid mass lesions were unclear and had incomplete capsules.

Microscopic analysis features. Lesions were mainly composed of lymphoid hyperplasia and lymphoid follicles and had enlarged germinal centers. They were characterized by capillary proliferation and inflammatory changes, with marked eosinophil infiltration. In addition, eosinophilic microabscesses were observed. As compared with focal nodular lesions, diffuse mass lesions contained more fibrillar components and less vascular hyperplasia. Nodular lesions were characterized by enhanced vascular hyperplasia and fibrillar components. Nodular lesions revealed a large number of lymphoid follicles and enlarged germinal centers (Fig. 6). A large number of capillary hyperplasia with eosinophilic infiltration was observed in Fig. 7 and in Fig. 8 nodular lesions demonstrated more fibrillar components with eosinophilic infiltration. 


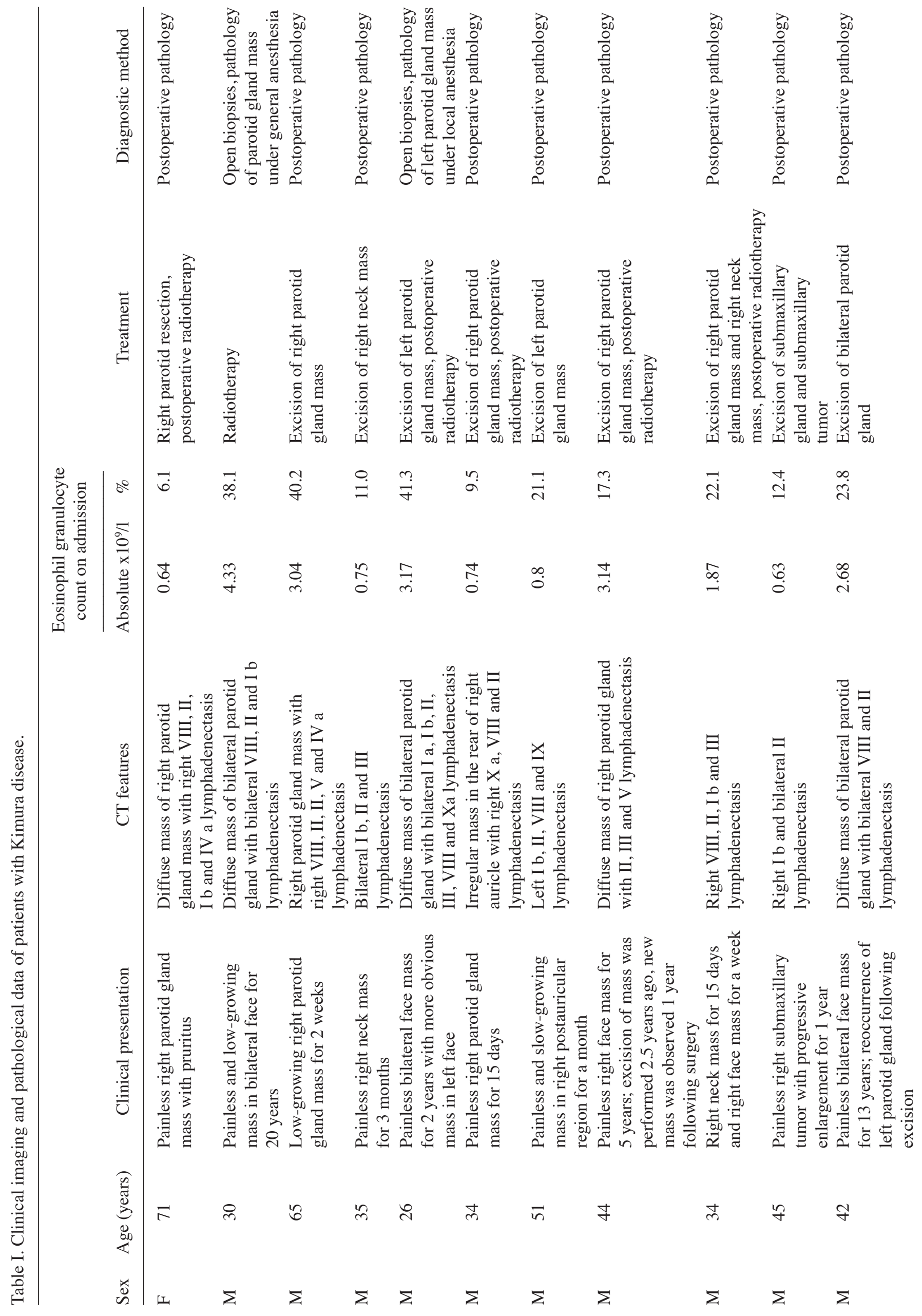




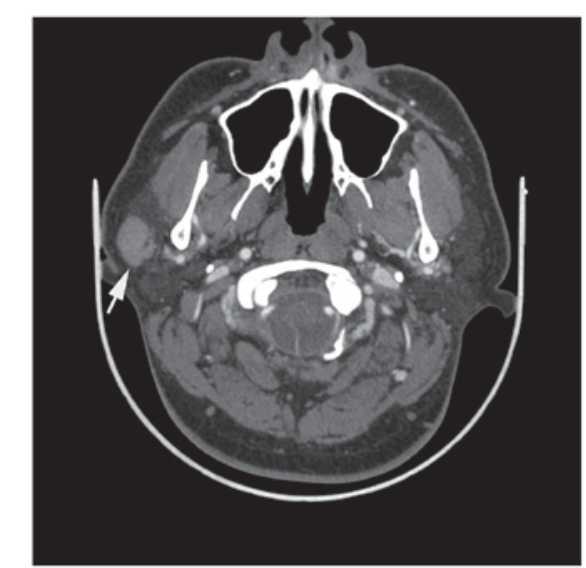

Figure 1. Computerized tomography scan of a patient (male, 34 years) with nodular-type Kimura disease in the right parotid gland. Soft tissue nodule indicated with an arrow and visible clear boundary, clear subcutaneous fat layer and marked homogeneous enhancement.

\section{Discussion}

Kimura disease is a rare lymphoproliferative disease of unknown origin. Kimura disease is characterized by lymphoproliferative formation of lymphoid follicles and eosinophilic granulomas in soft tissue (11). Kimura disease may occur at any age, although the peak age is $20-40$ years; the majority of cases occur in middle-aged males $(12,13)$. The male to female ratio has been reported to be as high as 14:1 (14). Kimura disease primarily occurs in head and neck regions and may affect major salivary glands and lymph nodes, especially in the parotid region $(15,16)$. There are a few reports of Kimura disease cases involving the groin, limbs, eyelid, tongue, auricle, hard palate or throat $(17,18)$. The onset age (middle aged, 35-50 years), sex (>90\% males) and site of pathological changes, with a majority in the head and neck regions potentially affecting major salivary glands and lymph nodes, in the current study were consistent with the literature $(12,13)$.

Ultrasound-guided core needle biopsy increased specimen adequacy and diagnosis to reduce the need and dependence on an onsite cytopathologist (19). For Kimura disease, magnetic resonance (MR) imaging is useful for determining lesion morphology, anatomical distribution, enhancement pattern and degree of intralesional vascularity (20). It has been reported that well-defined nodular masses or ill-defined plaque-like infiltrative masses from CT scans are often associated with lymphadenectasis (21). A majority of lesions is observed in the parotid gland (22). On MR images, masses exhibited variable signal intensity and visualized vascular structures (10). In the current study, $25.0 \%$ of patients (3/12) had bilateral involvement, which differed from the prevalence of bilateral involvement in previous report $(23,24)$.

Diagnosis of Kimura disease is based on the analysis of pathological specimens. Pathological features of Kimura disease include lymphoid tissue hyperplasia; lymphoid follicle formation; an active follicular germinal center; hyaline degeneration; infiltration of tissue, plasma and mast cells; and lymphatic sinus fibrosis $(25,26)$. Characteristic changes include infiltration of mature eosinophils in follicular, capsular and extracapsular regions and formation 

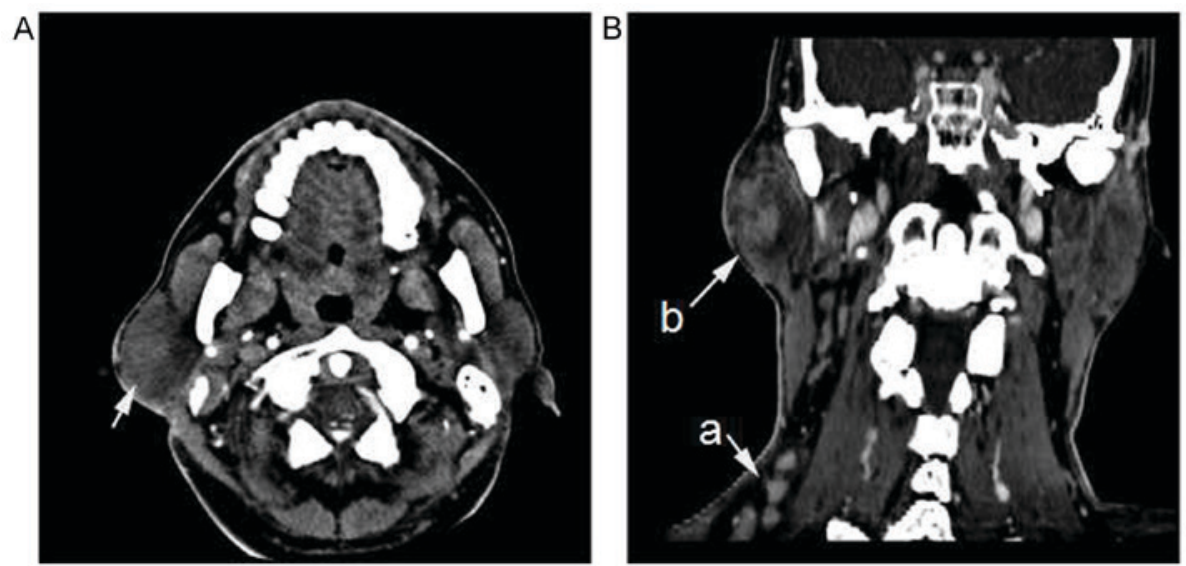

Figure 2. Computerized tomography scan of a patient (male, 44 years) with diffuse mass-type Kimura disease in the right parotid gland. (A) Right parotid gland swelling, disappearance of subcutaneous fat and mild or moderate heterogeneous enhancement of mass indicated with an arrow. (B) Enlarged lymph nodes are indicated with arrow ' $\mathrm{a}$ ' in the right neck, present a clear boundary and homogeneous enhancement. The enhancement was greater than that of the parotid lesion mass as indicated with the arrow ' $b$ '.

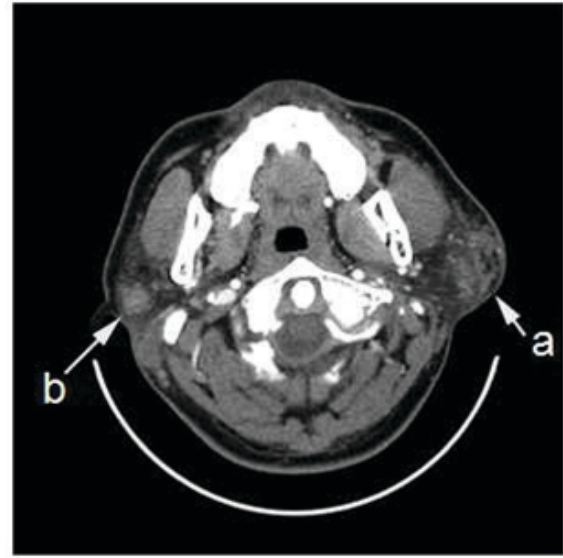

Figure 3. Computerized tomography scan of a patient (male, 42 years) with Kimura disease in the parotid gland. Bilateral nodules and a diffuse mass are visible. Right parotid gland nodular lesions presented no clear boundaries and marked homogeneous enhancement, as indicated with the arrow 'b'. The left parotid gland diffuse mass exhibited heterogeneous enhancement. Soft tissue of adjacent skin was thickened and the density of internal of subcutaneous fat was increased, highlighted with the arrow ' $a$ '.

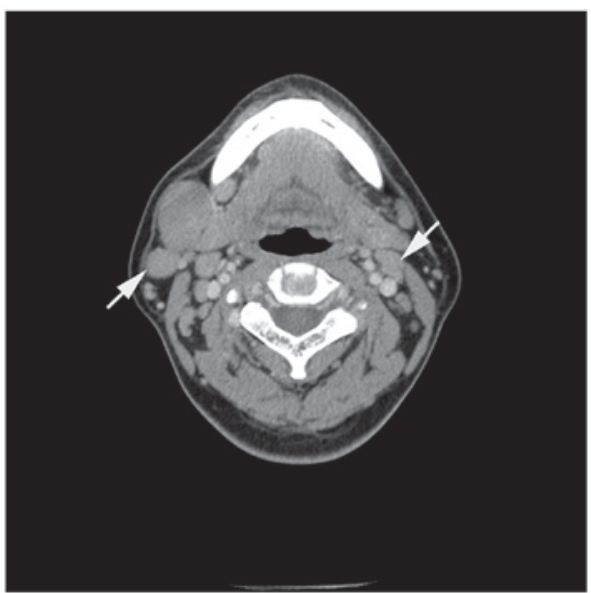

Figure 4. Computerized tomography scan in bilateral view of a swollen lymph node in the jaw and neck gap region, as indicated with an arrow. The boundaries of the lesions were clear, with homogeneous enhancement. There was no marked liquefaction necrosis, calcification or fusion.

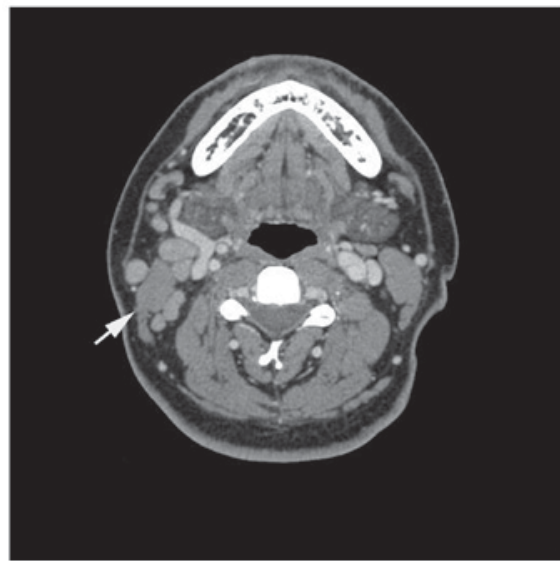

Figure 5. Computerized tomography scan in unilateral view of a swollen lymph node in the jaw and neck gap region, as indicated with an arrow. The boundaries of the lesions were clear, with homogeneous enhancement. There was no marked liquefaction necrosis, calcification or fusion.

of eosinophilic microabscesses (27). Takeishi et al (28) reported that a majority of neck lumps consisted of fibrous tissue hyperplasia in patients with long disease course. As the degree of enhancement is reduced in the presence of fewer vessels in neck region, the current study speculated that different degrees of enhancement in patients with Kimura disease may be associated with the degree of proliferation of fibrous tissue and blood vessels. In a follow-up study of patients with eosinophilic lymphogranulomas neck nodules, Lim et al (29) reported that lesion-enhanced areas exhibited a trend toward gradual narrowing. Such narrowing may be associated with fibrosis and sclerosis of postcapillary venules. In the present study, CT and pathological analysis revealed capillary proliferation within lesions in patients with a short disease course. It was considered that this proliferation may be the pathological basis of homogeneous enhancement of the lesions. Capillary proliferation was not observed in patients with a long disease course ( $\geq 10$ years). Interstitial fibrosis and hyaline degeneration of the capillary wall were further observed. These features may be the pathological 
basis of mild to moderate enhancement in head and neck lesions. Dynamic enhanced CT revealed characteristics of progressive strengthening, indicating vascular proliferation and fiber components in lesions. In 1 patient with neck lymph and parotid gland involvement, the degree and mode of enhancement were differed. In this patient, as the course of the disease progressed, the degree of vascular proliferation and interstitial fibrosis in the parotid gland and lymph nodes were inconsistent. Further studies are required to clarify the underlying mechanisms.

There are many diseases of the parotid gland, including benign tumors, malignant tumors and inflammatory lesions, making a differential diagnosis of parotid gland nodules difficult. A careful analysis of clinical imaging features is necessary for an accurate diagnosis (29). Pleomorphic adenomas and adenolymphomas are the most common benign tumors. Pleomorphic adenomas are often single, with delayed enhancement (30). Adenolymphomas are usually found in elderly males. Adenolymphomas are often discovered during a superficial parotidectomy and exhibit marked enhancement in early stages. The density decreases rapidly in cases of delayed diagnosis and density may be heterogeneous in some cases (30). In the present study, low-density areas were observed in lesions. In addition, cervical lymph nodes were not swollen, which was different from low-density Kimura disease involving cervical lymph nodes. Diagnosis may be combined with clinical manifestations and elevated eosinophils in peripheral blood.

Hemorrhagic necrosis and cystic degeneration are common in lesions in primary malignant tumors of the parotid gland and neck metastases (31). In contrast, liquefaction necrosis is uncommonin Kimura disease.Bilaterallymphnodes are swollen in cases of lymphoma and lymph node fusion is frequently observed. It may be differentiated from Kimura disease, which exhibits a clear boundary and no marked fusion trend (32). In addition, patients with lymph node fusion, calcification and local necrosis present general symptoms, including fever and fatigue (33). Using a combination of clinical examinations and molecular laboratory tests, Kimura disease may be identified more easily.

According to previous research, Kimura disease outcomes are positive following comprehensive treatment, which may consist of surgery, radiotherapy and adrenal hormone therapy (34). Diagnosis of Kimura disease strongly relies on pathology and imaging features. Kimura disease is commonly found in head and neck regions of middle-aged males, who exhibit a painless mass, with or without itchy skin and pigmentation, in addition to an increased peripheral blood eosinophil count.

Diagnosis of Kimura disease in head and neck regions may be improved based on lesions with clear or unclear boundaries, homogeneous or heterogeneous enhancement with or without lymphadenectasis and by the presence of peripheral blood eosinophilia. All of which describe the pathological basis for the diagnosis of Kimura disease. Future research aims to increase the number of patients to allow a more systematic approach for diagnosis of Kimura disease.

\section{Acknowledgements}

Not applicable.

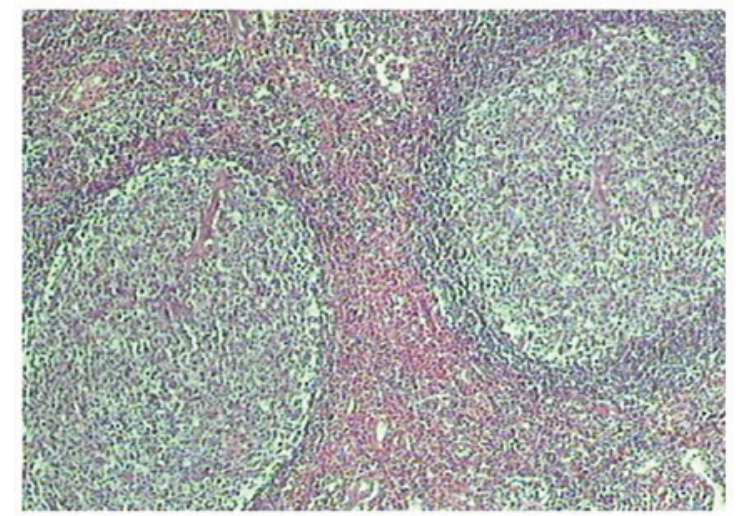

Figure 6. Hematoxylin-eosin stain highlighting the formation of lymphoid follicles and an enlarged germinal center (magnification, $\mathrm{x} 40$ ).

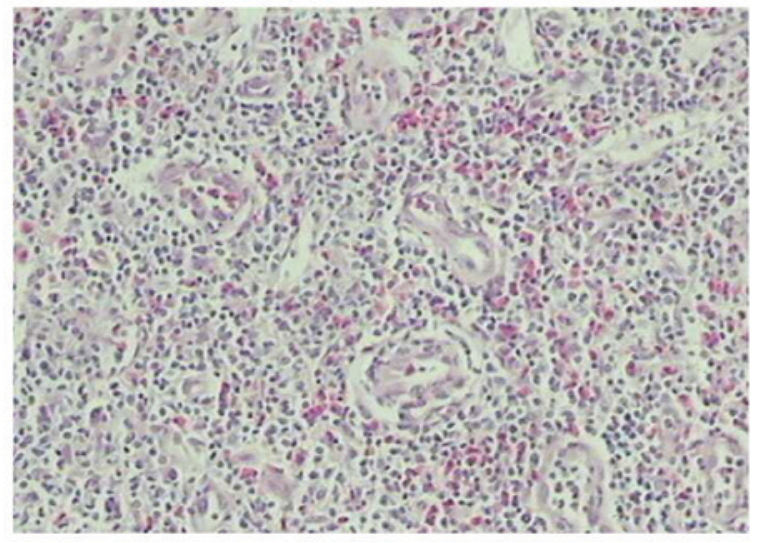

Figure 7. Hematoxylin-eosin stain highlighting the formation of capillary hyperplasia and eosinophil infiltration (magnification, $\mathrm{x} 100$ ).

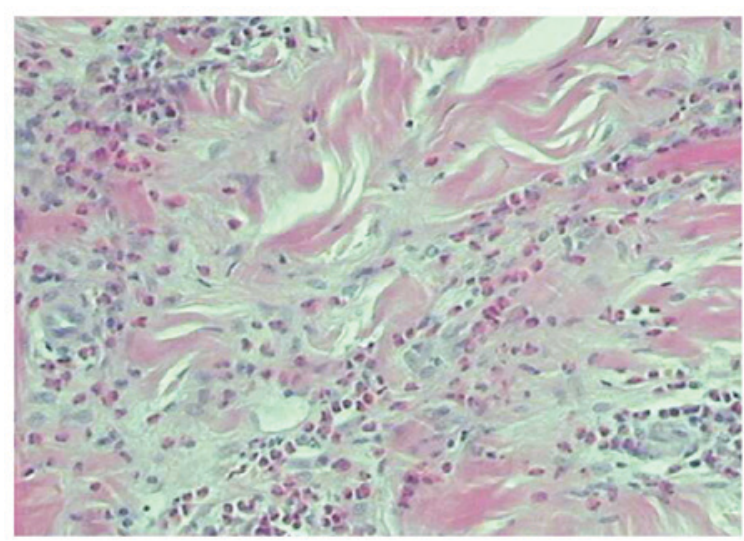

Figure 8. Hematoxylin-eosin stain highlighting interstitial fibrosis and eosinophil infiltration (magnification, x100).

\section{Funding}

No funding was received.

\section{Availability of data and materials}

The datasets used and/or analyzed during the current study are available from the corresponding author on reasonable request. 


\section{Authors' contributions}

LZ and LY designed the study, recruited patients, analyzed data and drafted the manuscript. W-Z, J-NM and C-QZ collected the cases, analyzed the data and revised the manuscript. All authors reviewed and approved the final manuscript.

\section{Ethics approval and consent to participate}

All experiments were approved by the Ethics Committee of Cangzhou Central Hospital and all patients provided written informed consent.

\section{Patient consent for publication}

Not applicable.

\section{Competing interests}

The authors declare that they have no competing interests.

\section{References}

1. Gao Y, Chen Y and Yu GY: Clinicopathologic study of parotid involvement in 21 cases of eosinophilic hyperplastic lymphogranuloma (Kimura's disease). Oral Surg Oral Med Oral Pathol Oral Radiol Endod 102: 651-658, 2006.

2. Lanjewar DN, Bhosale A and Iyer A: Spectrum of dermatopathologic lesions associated with HIV/AIDS in India. Indian J Pathol Microbiol 45: 293-298, 1996.

3. de Castro HA Jr, Lasmar MT, de Souza EA, Figueiredo JA, Nogueira BD and Tardelli FC: Renal epitelial neoplasia associated with Kimura disease. Arch Esp Urol 63: 547-549, 2010 (In English, Spanish).

4. Nonaka M, Sakitani E, Ono E, Yamamura Y, Seo Y, Shibata N, Pawankar R and Yoshihara T: Basophils are increased and express increased levels of interleukin- 4 in the parotid lesions of kimura disease. Asia Pac Allergy 7: 221-226, 2017.

5. Nonaka M, Sakitani E and Yoshihara T: Anti-IgE therapy to Kimura's disease: A pilot study. Auris Nasus Larynx 41: 384-388, 2014.

6. Gopinathan A and Tan TY: Kimura's disease: Imaging patterns on computed tomography. Clin Radiol 64: 994-999, 2009.

7. Jiang Y, Hua Q, Ren J, Zeng F, Sheng J, Liao H, Zhang Z and Guan H: Eosinophilic hyperplastic lymphogranuloma: Clinical diagnosis and treatment experience of 41 cases. Am J Otolaryngol 38: 626-629, 2017.

8. Matsuo T, Tanaka T and Kinomura M: Nephrotic syndrome during the tapering of oral steroids after pathological diagnosis of Kimura disease from a lacrimal gland mass: Case report and review of 10 Japanese patients. J Clin Exp Hematop 57: 147-152, 2017.

9. Zhang R, Ban XH, Mo YX, Lv MM, Duan XH, Shen J, Li JP, Liu XW and Xie CM: Kimura's disease: The CT and MRI characteristics in fifteen cases. Eur J Radiol 80: 489-497, 2011.

10. Lin YY, Jung SM, Ko SF, Toh CH, Wong AM, Chen YR, Chan SC, Cheung YC and Ng SH: Kimura's disease: Clinical and imaging parameters for the prediction of disease recurrence. Clin Imaging 36: 272-278, 2012

11. Wang DY, Mao JH, Zhang Y, Gu WZ, Zhao SA, Chen YF and Liu AM: Kimura disease: A case report and review of the chinese literature. Nephron Clin Pract 111: c55-c61, 2009.

12. Chen C, Chen K, Huang X, Wang K and Qian S: Concurrent eosinophilia and IgG4-related disease in a child: A case report and review of the literature. Exp Ther Med 15: 2739-2748, 2018.

13. Iguchi Y, Inoue T, Shimono M, Yamamura T, Shigematsu T and Takahashi S: Kimura's disease and its relation to angiolymphoid hyperplasia with eosinophilia: Report of three cases and review of the literature. J Oral Pathol 15: 132-137, 1986.

14. Arshad AR: Kimura's disease of parotid gland presenting as solitary parotid swelling. Head Neck 25: 754-757, 2003.
15. Sawaimul K, Iqbal B and Kambale T: Kimura's disease embedding radial artery: A very rare presentation. J Cancer Res Ther 11: 1031, 2015.

16. Ye P, Wei T, Yu GY, Wu LL and Peng X: Comparison of local recurrence rate of three treatment modalities for kimura disease. J Craniofac Surg 27: 170-174, 2016.

17. Wang $\mathrm{H}$ and Zheng $\mathrm{Z}$ : One case of parotid eosinophilic lymphogranuloma. Lin Chung Er Bi Yan Hou Tou Jing Wai Ke Za Zhi 28: 830-831, 2014 (In Chinese).

18. Zhang JZ, Zhang CG and Chen JM: Thirty-five cases of Kimura's disease (eosinophilic lymphogranuloma). Br J Dermatol 139: 542-543, 1998

19. Koh H, Kamiishi N, Chiyotani A, Takahashi H, Sudo A, Masuda Y, Shinden S, Tajima A, Kimura Y and Kimura T: Eosinophilic lung disease complicated by Kimura's disease: A case report and literature review. Intern Med 51: 3163-3167, 2012.

20. Naveed M, Siddiqui AA, Kowalski TE, Loren DE, Khalid A, Soomro A, Mazhar SM, Yoo J, Hasan R, Yalamanchili S, et al: A Multicenter comparative trial of a novel EUS-guided core biopsy needle (SharkCore) with the 22-gauge needle in patients with solid pancreatic mass lesions. Endosc Ultrasound 7: 34-40, 2018.

21. Baba A, Ojiri H, Dogru M, Tanaka Y, Takahashi S, Mogami T, Kobashi Y, Yamazoe S, Nozawa Y, Ogino N, et al: An unusual clinical presentation of kimura disease manifesting with a typical cephalocervical lesion and an atypical subcutaneous hip mass lesion. Intern Med 55: 1017-1020, 2016.

22. Wang J, Tang Z, Feng X, Zeng W, Tang W, Wu L and Jin L: Preliminary study of diffusion-weighted imaging and magnetic resonance spectroscopy imaging in Kimura disease. J Craniofac Surg 25: 2147-2151, 2014.

23. Park SW, Kim HJ, Sung KJ, Lee JH and Park IS: Kimura disease: $\mathrm{CT}$ and MR imaging findings. AJNR Am J Neuroradiol 33: 784-788, 2012.

24. Iwai H, Nakae K, Ikeda K, Ogura M, Miyamoto M, Omae M, Kaneko T and Yamashita T: Kimura disease: Diagnosis and prognostic factors. Otolaryngol Head Neck Surg 137: 306-311, 2007.

25. Kuroda K, Kashiwagi S, Teraoka H, Kinoshita H, Nanbara M, Noda E, Chikugo T, Hirakawa K and Ohira M: Kimura's disease affecting the axillary lymph nodes: A case report. BMC Surg 17: 63, 2017.

26. Uysal IO, Eryilmaz MA, Salk I and Abasiyanik F: Kimura disease in the parotid gland. J Craniofac Surg 22: 337-338, 2011.

27. Xu ZF, Yong F, Yu T, Chen YY, Gao Q, Zhou T, Pan AZ and Wu RH: Different histological subtypes of parotid gland tumors: CT findings and diagnostic strategy. World J Radiol 5: 313-320, 2013.

28. Takeishi M, Makino Y, Nishioka H, Miyawaki T and Kurihara K: Kimura disease: Diagnostic imaging findings and surgical treatment. J Craniofacial Surg 18: 1062-1067, 2007.

29. Lim WE, Tan NG and Tan KP: Radiological features in a patient with Kimura's disease. Singapore Med J 38: 125-128, 1997.

30. Bastos JT, Rocha CRMD, Silva PMCE, Freitas BMP, Cassia FF and Avelleira JCR: Angiolymphoid hyperplasia with eosinophilia versus Kimura's disease: A case report and a clinical and histopathological comparison. An Bras Dermatol 92: 392-394, 2017.

31. Lu L, Chen RG, Li XQ and Wang J: Kimura disease and epithelioid hemangioma: A comparative study of 12 cases. Zhonghua Bing Li Xue Za Zhi 34: 353-357, 2005 (In Chinese).

32. Mantsopoulos K, Goncalves M, Koch M, Iro H and Agaimy A: Submandibular gland pleomorphic adenoma: Histopathological capsular characteristics and correlation with the surgical outcome. Ann Diagn Pathol 34: 166-169, 2018.

33. Yu S, Zhang Z, Bao Q, Su J, Liu M, Shi Q and Cai W: Diffusion kurtosis imaging in the differential diagnosis of parotid gland disease and parotid adenolymphoma: Preliminary results. Dentomaxillofac Radiol 16: 20170388, 2018.

34. Chen Y, Wang J, Xu F, Zeng C and Liu Z: Clinicopathological features and prognosis of kimura's disease with renal involvement in Chinese patients. Clin Nephrol 85: 332-339, 2016. 